\title{
FE model of reticulated dome under impact based on the MJ-C
}

\author{
L.LIN ${ }^{1,2}$, Y.H. Sun ${ }^{1} \&$ M.Y. Huang ${ }^{1} \&$ J. Li ${ }^{1} \&$ X.Q.Song ${ }^{1} \&$ Z.X.Duan ${ }^{1} \&$ X.F. \\ Huang1,*
}

1,College of Civil Engineering and Architecture, Harbin University of Science and Technology, Harbin, PR China

2,School of Civil Engineering, Harbin Institute of Technology, Harbin, PR China

1,College of Civil Engineering and Architecture, Harbin University of Science and Technology, Harbin, PR China

\begin{abstract}
KEYWORD: K8 single-layer reticulated dome; Impact load; MJ-C; SHELL163; Temperature effect ABSTRACT: A large number of research results show that the accuracy of the material model largely determines the accuracy of the numerical simulation results. In this paper, the model of Kiewitt8 single-layer reticulated dome with a span of $40 \mathrm{~m}$ is established by ANSYS/LS-DYNA. The difference between the model and others are that the Modified Johnson-Cook (MJ-C) constitutive model and fracture initiation are used for the material model of main rods, ball nodes and roof panel, and the main rods and ball nodes are modeled with a shell element, SHELL163. The mechanical behaviors of structural materials under the influence of large strain, high strain rate and temperature are considered in this model, and the mechanical response of the structure under impact can be described more accurately. The paper mainly solves the problem of modeling and analyses the results of the numerical simulation, lays a foundation of further study on the performance of reticulated dome under impact.
\end{abstract}

\section{INTRODUCTION}

The performance of the whole building structure subjected to impact was paid more attention to after the "911" incident. For the reason of the particularity of impact load, the numerical simulation analysis is one of the most common methods. At present, the piecewise linear plasticity model is used for the majority of material models on the numerical simulation study of impact resistance of reticulated shell structures. For example, in Harbin Institute of Technology, Wang Duozhi and other scholars built the numerical model of Kiewitt8 single-layer reticulated dome with a span of $60 \mathrm{~m}$ and studied its failure types and properties under impact load ${ }^{[1][2]}$. Lin $\mathrm{Li}$ et al established the numerical model of the Kiewitt8 single-layer reticulated dome under the impact of aircraft, and analyzed the failure mode ${ }^{[3]}$. In Lanzhou University of Technology, Wang Xiuli and other scholars used the material model to establish a single-layer reticulated shell with lower steel pipe column, and studied the dynamic response of the structure under impact load ${ }^{[4]}$. This material model is multi-linear elastic-plastic material model, can enter the stress strain curve associated with strain rate, but the effect of temperature in the process of impact hasn't been consider. Therefore, the JohnsonCook (J-C) material model has been used for the research of components under impact, and the results of numerical simulation were excellent. Such as Gupta and other scholars studied the projectile penetration behavior of thin aluminum target ${ }^{[5]}$, used the $\mathrm{J}-\mathrm{C}$ constitutive model and $\mathrm{J}-\mathrm{C}$ fracture initiation to simulate the target experiment by the establishing two-dimensional axisymmetric model, and a good result was achieved comparing with the experiment; Iqbal and Gupta ${ }^{[6]}$ established three dimensional numerical model, used the $\mathrm{J}-\mathrm{C}$ constitutive model and $\mathrm{J}-\mathrm{C}$ fracture initiation to simulate the experiment in [5], and the failure mode was in accordance with the experiment. Zhou and Stronge ${ }^{[7]}$ used ABAQUS /EXPLICIT to establish a two-dimensional axisymmetric model with the J-C strength equation and the cumulative failure initiation, and the simulation results are consistent with the experimental results. The writer adopted a series of research methods, obtained the ten parameters of the J-C material model for the Q235B steel which was widely used in the Chinese reticulated domes. A mainly modification was done on the temperature softening of the yield 
strength and the nonlinear temperature softening effect of the ductility. Eventually, the MJ-C constitutive equation (1) and fracture initiation equation (2) were got :

$\sigma_{\text {eq }}=\left(\mathrm{A}+\mathrm{B} \varepsilon^{\mathrm{n}}\right.$ eq $)\left(1+\mathrm{Cln} \varepsilon^{*}{ }_{\text {eq }}\right)\left(1-\mathrm{FT}^{* \mathrm{~m}}\right)$

$\varepsilon \mathrm{f}=\left[\mathrm{D}_{1}+\mathrm{D}_{2} \exp \left(\mathrm{D}_{3} \sigma^{*}\right)\right] \times\left(1+\mathrm{D}_{4} \ln \varepsilon^{*}{ }_{\mathrm{eq}}\right)\left[1+\mathrm{D}_{5} \exp \left(\mathrm{D}_{6} \mathrm{~T}\right.\right.$

$*)]$

Type: A, B, n, C, F, m - the model parameter,

$\mathrm{D}_{1} \sim \mathrm{D}_{6}$ - the material constant,

$\sigma_{\text {eq }}$ - equivalent stress,

$\varepsilon$ eq - equivalent strain,

$\dot{\varepsilon}^{*}$ eq - the dimensionless equivalent plastic strain rate, $\dot{\varepsilon}^{*}$ eq $=\dot{\varepsilon}_{\mathrm{eq}} / \dot{\varepsilon}_{\mathrm{o}}, \dot{\varepsilon}_{\mathrm{o}}$ is reference strain rate,

$\mathrm{T}^{*}$ - the dimensionless temperature, $\mathrm{T}^{*}=(\mathrm{T}-\mathrm{Tr}) /(\mathrm{Tm}-\mathrm{Tr}), \mathrm{Tr}$ is reference temperature, $\mathrm{Tm}$ is the melting point of materials, $\mathrm{T}$ is the current temperature,

$\varepsilon f$ - the fracture strain,

$\sigma^{*}$ - triaxial stress degree, $\sigma^{*}=\sigma H / \sigma e q, \sigma H$ is Average stress,

The three parts on the right side of equation(1) represent the equivalent plastic strain, strain rate and temperature effects on the flow stress ( specific parameters are shown in Table 1)[8]. In this paper, the numerical model of Kiewitt8 single-layer reticulated dome is established by this material model. Table 1. MJ-C parameters ${ }^{[8]}$

\begin{tabular}{cccccc}
\hline $\begin{array}{c}A \\
(\mathrm{MPa})\end{array}$ & $\begin{array}{c}B \\
(\mathrm{MPa})\end{array}$ & $n$ & $C$ & $F$ & $m$ \\
\hline 244.8 & 899.7 & 0.940 & 0.0391 & 1.989 & 0.1515 \\
\hline$D_{1}$ & $D_{2}$ & $D_{3}$ & $D_{4}$ & $D_{5}$ & $D_{6}$ \\
\hline-43.408 & 44.608 & -0.016 & 0.0145 & -0.046 & 7.776 \\
\hline
\end{tabular}

\section{NUMERICAL MODEL}

As the main structural member in the reticulated domes, the modeling of main rod and node is the key process. Dealing with the connection between them is the important step in the process of modeling. In addition, roof panel and other non-structural members cannot be ignored. The key steps of building the Kiewitt8 single-layer reticulated dome with roof panel are introduced next.

\section{Structural style}

As shown in Figure 1, select the Kiewitt8 single-layer reticulated dome. The length of span (L) is $40 \mathrm{~m}$, and rise span ratio ( $\mathrm{f} / \mathrm{L}$ ) is $1 / 5$. The number of the loop members is 6 , running from the first loop at the apex down to the sixth loop to the outer perimeter. The main rods of the structure are divided into the longitudinal main rib, the ring rod and the inclined rod. (The specific parameters of the rods and nodes are shown in Table 2). Thickness of the roof panel is $0.002 \mathrm{~m}$; distributed load is $120 \mathrm{~kg} / \mathrm{m}^{2}$; the impact velocity and quality are changeable. In the impact process, the impact object is regarded as a rigid body.

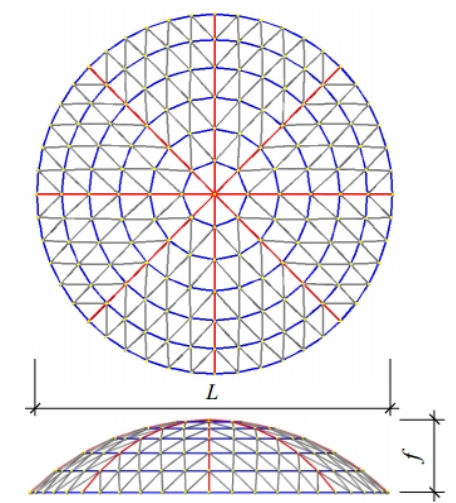

Figure 1. The structure schematic diagram of Kiewitt8 single-layer reticulated dome 
Table 2. rod and node parameter (mm)

\begin{tabular}{lcccc}
\hline & Main rib & Loop rod & $\begin{array}{c}\text { Inclined } \\
\text { rod }\end{array}$ & Ball node \\
\hline Diameter & 121 & 121 & 114 & 500 \\
\hline Thickness & 3.5 & 3.5 & 3.0 & 30 \\
\hline
\end{tabular}

\section{The numerical model of the main rod and node}

In ANSYS/LS-DYNA, there are 9 kinds of dynamic analysis units: LINK160, BEAM161, PLANE162, SHELL163, SOLID164, COMBI165, MASS166, LINK167 and SOLID168. In this paper, the simulation object is Kiewitt8 single-layer reticulated dome, the material of the main rod is thin-walled seamless steel tube whose cross section is a circular ring shape, and the ball node is hollow sphere. The geometric features of the main rod not only are in line with the characteristics of BEAM161 (which is the scale of a direction is much larger than the scale of the other two directions), namely the length of pipe is much more than the height and width of the section; but also are in line with the characteristics of SHELL163 (which is the scale of a direction is far less than the scale of the other two directions), namely the thickness of steel pipes is far less than the height and length of the section. Yet BEAM161 unit will not be able to choose the MJ-C material model, so the main rod is established with SHELL163 element. Similarly, ball nodes also choose SHELL163 element.

SHELL163 is a 4-node element with both bending and membrane capabilities. Both in-plane and normal loads are permitted. The element has 12 degrees of freedom at each node: translations, accelerations, and velocities in the nodal $\mathrm{x}, \mathrm{y}$, and $\mathrm{z}$ directions and rotations about the nodal $\mathrm{x}, \mathrm{y}$, and z-axes (See Figure 2) [9].

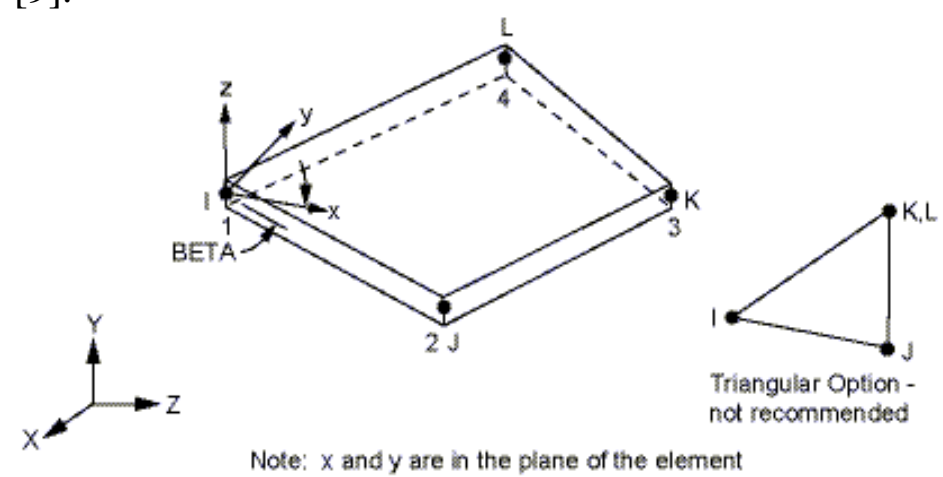

Figure 2. The geometric diagram of SHELL163 element ${ }^{[9]}$

The operation of command flow is adopted in the whole process of numerical modeling. After the key points are established according to their geometric coordinates, the 169 sphere models is established by the conversion of local coordinate system and the "sphere" command in ANSYS in the location of the key points. Then the ball is deleted, but the elements whose dimension is lower than volume are reserved. So that the geometric modeling of the ball nodes is completed (see Figure 3 ).

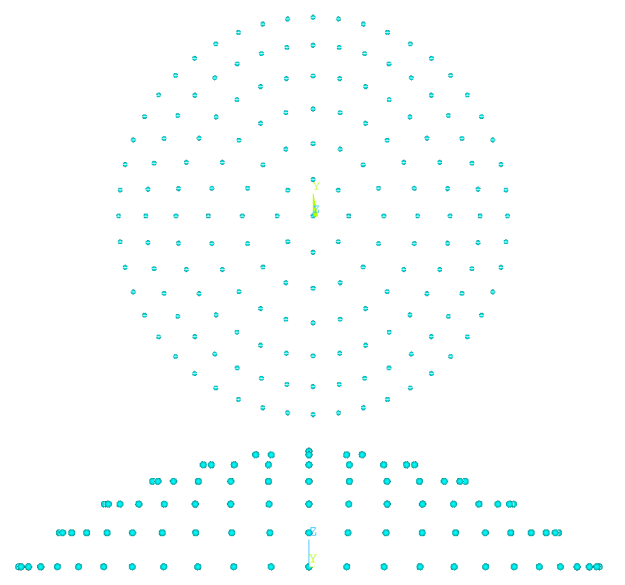

Figure 3. Schematic diagram of ball nodes 
The the main rod is established in the form of the cylindrical surface. The "ADRAG" and "AROTAT" command are usually selected. "ADRAG" command is limited by the number of drag lines which is round, and a rod can be composed of six surfaces at most. The establishment is complex, and the operation of connection command between the link and the node is inconvenient. The last parameter "NSEG" of the "AROTAT" command can be set freely, which is beneficial to the gridding division and the operation of the subsequent commands (see Figure 4). Therefore, the model finally uses the "AROTAT" command, and "NSEG" value takes 10. In this way, a total of 456 link units are completed. It should be noticed that the key points which are required in modeling main rods should be established referencing to the original key points before the "AROTAT" command. In other words, the radius of the rod is added to the $\mathrm{Z}$ axis coordinate values of the original key points, the value of $\mathrm{X}$ and $\mathrm{Y}$ axis values are unchanged.

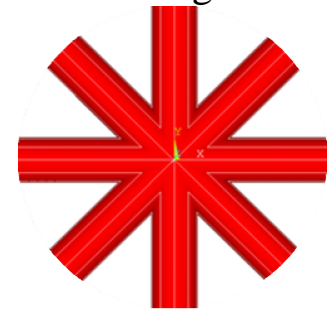

Figure 4. Intersection of local rods at the apex of dome

\section{Connection of main rod and ball node}

The geometric relation between the rod and the node is processed by Boolean operation before dealing with the connection problem between the rod and the node. In view of this model, we need to delete the redundant rod in the ball node, so we use the Boolean subtraction algorithm. The ball node surface minus the rod surface, so that the rod is divided into two parts (see Figure 5), and then cut out the rods of the intersection in the ball nodes, geometric relations will be dealt with (see Figure 6). Don't use the rod surface minus the surface of ball node, it need be paid more attention to. If do as this , the ball node surface will be cut hive off some of the small circle planes, and ball nodes with holes are not conducive to the later gridding division operation. After that, the Boolean operation can be continually used for the connection processing of the link and node, the set of the tolerance with the "AGLUE" command, and divide the unit. But there may be deformed unit, so this method isn't adopted. After the gridding division, set the tolerance, using the "NUMMRG" command to connect the rod and the node together. Because the geometric model is regular, the gridding division is relatively easy. The ball shell need be cut into 8 parts by the working plane before the ball node division, and then divide the ball.
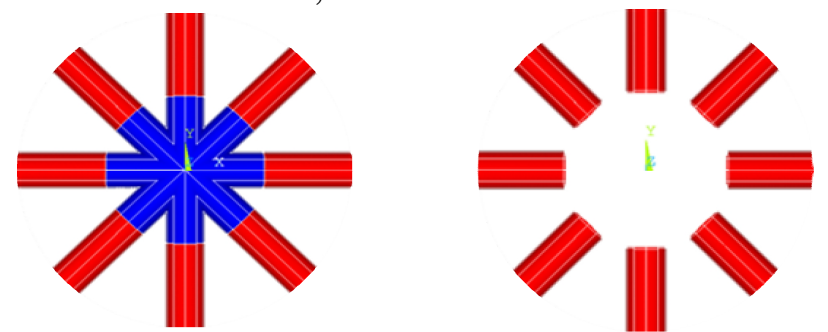

Figure 5. The local rods Figure 6. The local rods graph after Boolean opera- graph after deleting untion necessary parts

\section{Numerical model of roof panel}

Finally, the MJ-C model and the SHELL163 element are selected to establish the numerical model of the roof panel. The location of the key points of the roof panel need be determined at first, and then the triangular roof panel area is formed by the key points in accordance with the geometric features of the roof panel. The roof panel connects the main rods and nodes with the purlin and supporting purlin. After gridding division, the connection between the overall structural components is completed using the "NUMMRG" command. The dome is simply supported along the perimeter. The impact object which is built by using SOLID164 element and "CYLIND" command is a cylin- 
drical rigid body. Its diameter is 5 meters, the height is 2 meters. The final model is shown in Figure 7.

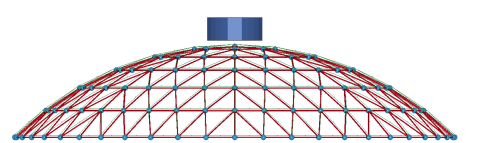

a. the dome structure without roof panel

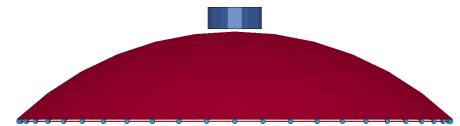

b. the dome structure with roof panel

Figure 7. Whole structure model.

The $\mathrm{K}$ file of the numerical model established by MJ-C material model is about $46 \mathrm{MB}$, and $\mathrm{K}$ file at past size is about $2 \mathrm{MB}$, so it puts forward higher requirements on the computer. With the development and the progress of modern computer, it provides a basis for modeling and calculation.

\section{RESULT ANALYSIS}

According to the plastic development and the vertical displacement of reticulated dome under the impact load, five failure modes were identified ${ }^{[10]}$ :

Dome is not penetrated. Mode 1: intact. Mode 2: slight damage.

Dome is penetrated. Mode 3: moderate damage; Mode 4: serious damage. Mode 5: global collapse. The impactor hits the apex of dome and the apex node is broken at the $0.008 \mathrm{~s}$, and the members around the apex are damaged. At the $0.050 \mathrm{~s}$, the rods and nodes within the 2nd loop are damaged. And, the $3 \mathrm{rd}$ loop is damaged at the $0.075 \mathrm{~s}$, the 4 th loop is damaged at the $0.199 \mathrm{~s}$. At the $0.449 \mathrm{~s}$, parts of the rods and nodes within the 5th loop are damaged. At the 0.500s, few rods and nodes within the 6th loop which separate from the structure are damaged.

The node displacements of each loop are shown in Figure 8. The displacements of the 1st, 2nd and 3rd loop are larger, and the change of displacement of the 2nd loop is the largest. The displacement of the 4th, 5th and 6th loop all have the upward trend around $0.15 \mathrm{~s}$, and then decline, however the change is small. Therefore, the numerical model belongs to the fifth kind of failure mode.

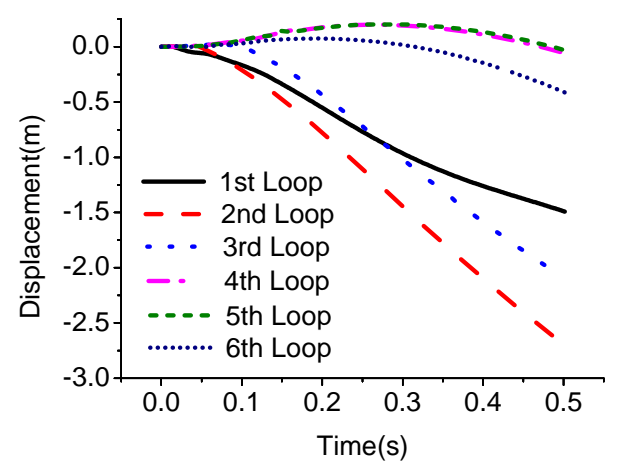

Figure 8. Displacement of the node within each loop.

\section{CONCLUSION}

In this paper, the numerical model of Kiewitt8 single-layer reticulated dome with a span of $40 \mathrm{~m}$ is established by ANSYS/LS-DYNA. Main rods and nodes are built by the MJ-C material model and shell163 elements. And the temperature effect is considered in spite of $\mathrm{K}$ file is more than 20 times of the model of the K file established with BEAM161 element. The calculation results of the model under the impact of rigid impactor whose velocity is $85 \mathrm{~m} / \mathrm{s}$ and the mass is $25,000 \mathrm{~kg}$ are analyzed, a conclusion is drawn that the failure mode of the whole structure is global collapse. 


\section{ACKNOWLEDGMENTS}

This work has been conducted with the financial support from the science and technology research projects of the Heilongjiang Province Natural Science Foundation of China (E201438); The Education Department of Heilongjiang province (12531135); The National Natural Science Foundation of China (project designation: 51378164 and 50978077)

\section{REFERENCE}

[1] Wang Duozhi, Zhi Xudong, Fan Feng, Shen Shizhao. Failure patterns of Kiewitt8 single-layer reticulated domes under impact loads[J]. Engineering Mechanics, 2008, 25(Sup. II ): 144-149(in Chinese)

[2] Wang Duozhi, Fan Feng, Zhi Xudong, Shen Shizha. Performance of single-layer reticulated domes under impact load and gravity[J]. Journal of Harbin Institute of Technology, 2009, 41(8): 1923(in Chinese)

[3] Wang Xiuli, Zhang Fuqiang, Yang Wenwei, Ma Xiaotong. Dynamic Response analysis of single-layer spherical reticulated shell with sub steel column under impact load[J]. Journal of Architecture and Civil Engineering, 2015, 32(3): 21-27.(in Chinese)

[4] Lin Li, Refined study on impact response and failure of reticulated shells[D]. Harbin: Harbin Institute of Technology, 2015(in Chinese)

[5] Gupta NK, Iqbal MA, Sekhon GS. Effect of Projectile Nose Shape, Impact Velocity and Target Thickness on the Deformation Behavior of Layered Plates[J]. International Journal of Impact Engineering, 2008, 35(1): 37-60.

[6] Iqbal MA, Gupta NK. Ballistic Limit of Single and Layered Aluminium Plates[J]. Strain, 2011, 47(Sup. I ): e205-e219

[7] Zhou DW, Stronge WJ. Ballistic Limit for Oblique Impact of Thin Sandwich Panels and Spaced Plates[J]. International Journal of Impact Engineering. 2008, 35(11): 1339-1354.

[8] Lin Li, Zhi Xudong, Fan Feng, et al. Determinateion of parameters of Johnson-Cook models of Q235B steel[J]. Journal of Vibration and Shock, 2014, 33(9): 153-158. (in Chinese)

[9] Pera Global. ANSYS/LS-DYNA User's Guide[M]. Beijing: Pera Global Limited Company, 1999.(in Chinese)

[10] Wang Duozhi. Failure Mechanism of Reticulated Shells Under Impact. Harbin: Harbin Institute of Technology, 2010(in Chinese) 